\title{
Online leadership discourse in higher education: a digital multimodal
}

\section{discourse perspective}

Sabine Tan ${ }^{1}$, Bradley A Smith ${ }^{1}$, and Kay L O’Halloran ${ }^{1}$

${ }^{1}$ School of Education, Curtin University, Perth, Australia

\section{Corresponding author:}

Kay L. O'Halloran, School of Education (Building 501), Faculty of Humanities, Curtin University, Kent Street Bentley, Perth, Western Australia 6102.

Email: kay.ohalloran@curtin.edu.au

\section{Author contact details:}

Dr Sabine Tan

School of Education (Building 501)

Faculty of Humanities

Curtin University

Kent Street Bentley

Perth Western Australia 6102

Email: sabine.tan@curtin.edu.au

Phone: +61 (8) 92663277

Dr Bradley A. Smith

4/27 Stephen Street

Yarraville, Victoria 3013

Email: semiosmith@gmail.com

Phone: +61 (03) 96877606. 
Associate Prof Kay L. O’Halloran

School of Education (Building 501)

Faculty of Humanities

Curtin University

Kent Street Bentley

Perth, Western Australia 6102

Email: kay.ohalloran@curtin.edu.au

Phone: +61 (8) 92662182

Number of words (excluding Appendix and Author Biographies): 8,218

File-size: $5,573,182$ bytes (5.6 MB) 


\title{
Online leadership discourse in higher education: a digital multimodal discourse perspective
}

\begin{abstract}
As leadership discourses in higher education are increasingly being mediated online, texts previously reserved for staff are now being made available in the public domain. As such, these texts become accessible for study, critique, and evaluation. Additionally, discourses previously confined to the written domain are now increasingly multimodal. Thus, an approach is required that is capable of relating detailed, complex multimodal discourse analyses to broader sociocultural perspectives to account for the complex meaning making practices that operate in online leadership discourses. For this purpose, a digital multimodal discourse approach is proposed and illustrated via a small-scale case study of the online leadership discourse of an Australian university. The analysis of two short video texts demonstrates how a digital multimodal discourse perspective facilitates the identification of key multimodal systems used for meaning making in online communication; how meaning arises through combinations of semiotic choices (not individual choices); and how the results of multimodal discourse analysis using digital technology can reveal larger sociocultural patterns; in this case, divergent leadership styles and approaches as reflected in online discourse, at a time of immense change within the higher education sector.
\end{abstract}

\section{Keywords}

University leadership communication; online leadership discourse; collegial and managerial leadership; digital multimodal discourse perspective; multimodal analysis; social semiotics 


\section{Introduction: leadership discourse in the online age}

In line with the move towards online teaching and learning in higher education, senior leadership also are increasingly turning towards online media to communicate not only with staff and students but with the wider public. The public online mediation of leadership discourse means that this wider audience includes potential stakeholders worldwide, such as prospective students and staff, other institutions and funding and regulatory agents. The fact of leadership discourse being publicly mediated online means that such discourses become texts for study, opening up such leadership communication for critique and appraisal (by both internal and external stakeholders).

Importantly also, such digital texts are inherently multimodal. Multimodal discourse is communication that employs and integrates multiple semiotic resources such as language, gaze, gesture, and proxemics. For example, whilst visual resources such as page layout and font contribute to meaning in print, video texts also draw on cinematographic and sound techniques (e.g. camera angle, music) (cf. Jewitt 2014; O'Halloran 2011; Van Leeuwen 1999), whereas websites utilise further hypermodal affordances (cf. Lemke 2002). The analysis of such documents, however, can be complex and calls for new approaches that are capable of relating the analysis of multimodal phenomena to broader sociocultural aspects, including ('critical') discourse perspectives (cf. Fairclough 2005; Iedema 2001; Machin 2013; Van Dijk 2001). As Van Dijk (2001: 115) observes, for example,

...society may also be analysed in more local and more global terms, firstly at the level of interaction and situations and secondly at the level of groups, social organizations, organizations and institutions. The latter, social structure, may only be related to discourse in two ways: firstly through the social representations of social members about such social structures, and secondly through the instantiation of social structures (such as groups and organizations) through social actors, interactions and situations at the local, micro level. [...] Thus, CDA may be interested in macro notions such as power and 
domination, but their actual study takes place at the micro level of discourse and social practices.

In this article, a digital multimodal discourse perspective is presented, which combines social semiotic theory and (critical) discourse analysis with software-based techniques to relate the micro-level analysis of (online) multimodal discourses to broader contextual aspects, in this case, divergent leadership styles and approaches in higher education. The analysis of two short video texts demonstrates how a digital multimodal discourse perspective facilitates the identification of key multimodal systems used in the construction of meaning in online leadership discourse; how meaning arises through combinations of semiotic choices (not individual choices); and how the results of close multimodal discourse analysis using digital technology can reveal larger social structures, in this case, conflicting leadership styles and approaches at a time of immense change within the higher education sector.

In the next section relevant aspects of the context of university leadership and communication are discussed, followed by the case study of two multimodal video texts from an Australian university. The article concludes with some observations on the usefulness of a digital approach for multimodal discourse analysis, limitations of the present study and future directions for research.

\section{Context: leadership communication in higher education}

Higher education globally has been undergoing significant and ongoing change in recent decades, in response to pressures such as an increasingly globalised and marketised higher education sector, an increasingly broad and diverse range of students, changes in funding, an increasing focus on standards and quality, and the information technology revolution (e.g. Fullan and Scott 2009; Marginson 2010; Scott et al. 2010). Within such a 'volatile operating 
context' (Scott et al. 2010: 401) the role and nature of university leadership has become a growing focus of interest (e.g. Bryman 2007; Fullan and Scott 2009; Middlehurst and Elton 1992; O’Mullane 2011; Scott et al. 2010).

A dominant trend noted in university leadership, driven in large part by such pressures, is the tension between a traditional collegial model of academia and an increasingly managerial style of executive leadership, and the consequent 'erosion of collegiality’ (Burnes, Wend and By 2013: 912; cf. Bryman 2007; Middlehurst and Elton 1992; Tapper and Pafreyman 2010). According to Burnes, Wend and By (2013: 912) this has led to 'a distancing effect on the relationship between academics and senior managers within universities', as 'staff have felt increasingly removed and even cut off from the decisionmaking process' (cf. also Coates et al. 2010; Tapper and Palfreyman 2010). Bryman (2007: 707) notes in this respect that 'the leadership of internally motivated employees requires considerable care... leadership that undermines collegiality, autonomy and the opportunity to participate in decisions...is likely to be ineffective because it damages the commitment of academics'. Thus, the return to a collegial approach to university leadership is advocated, or as Burnes, Wend and By (2013: 920) propose, 'the reinvention of collegiality to fit the needs of twenty-first century universities' (cf. also Bryman 2007; Tapper and Palfreyman 2010).

Communication has been identified as a crucial component of effective leadership (e.g. Bryman 2007). Scholars within the 'communication-as-constitutive' tradition of organisational studies (e.g. Cooren et al. 2011; Blaschke, Frost and Fabian 2014; Taylor and Robichaud 2004) go further: as Cooren et al. (2011: 1150) explain, communication cannot be considered to be simply one of the many factors involved in organizing, and it cannot be merely the vehicle for the expression of pre-existing 'realities'; rather, it is the means by which organizations are established, composed, designed, and sustained... Organizations are... ongoing and precarious accomplishments realized, experienced, and identified primarily - if not exclusively - in communication processes. 
A key issue in the study of higher education communication, as elsewhere in the social sciences, is the challenge of integrating micro and macro levels of analysis (Blaschke, Frost and Fabian 2014; Kuhn 2012; Taylor 2011), that is, "how to "zoom" in on a conversation and then out to see it in context' (Taylor 2011: 1285). Taylor makes a relevant distinction between the 'conversation' and the 'metaconversation': while the former enables 'locally constituted organization', the latter is 'a conversation of conversations' through which the organizational identity emerges and is constituted (Taylor 2011: 1279). Blaschke, Frost and Fabian (2014: 713) similarly distinguish between '[a]uthoritative texts' that 'project particular conceptions of structure and dynamics', communicated at the macro-level through legal frameworks and regulatory guidelines, and 'distributed conversations' which enact organisational change at the micro-level through shifts in goals and values (Blaschke, Frost and Fabian 2014: 716). They argue that the study of micro-patterns in communication can 'provide a sound explanation' for the emergence of broader 'institutional logics' such as, for example, the transition from 'collegialism' to 'managerialism in higher education (Blaschke, Frost and Fabian 2014: 727), but note that '[u]nfortunately, there is little explanation' of everyday university leadership practices at the 'micro level of (inter)actions' (Blaschke, Frost and Fabian 2014: 712).

The digital multimodal approach presented in this paper addresses this gap by demonstrating how the micro-analysis of the multimodal discourse patterns in two video texts of online leadership discourse at an Australian university reveals larger 'institutional logics' as embodied, for example, in divergent leadership styles and approaches, in the context of a university in transition.

\section{Case Study: multimodal online leadership discourse in an Australian university}

\section{The data: a university in transition}


The Australian university whose online leadership discourses form the data for the study discussed here, Curtin University, presents an interesting case study. At the time of data collection, the university was undergoing a dramatic university-wide restructure, to transform from a teaching-focussed to a more research-focussed university, affecting all staff, academic and non-academic; while into this situation a new Vice Chancellor was inaugurated in early 2014. Meanwhile, a large-scale initiative, which endeavoured to determine the existing and desired values of staff within the university, was ongoing.

Two key domains were selected for study in the project from which the analysis discussed in this paper is drawn: (1) the 'Living our Values' initiative - a strategic initiative that 'aims to foster an environment in which values play an important role in the decisionmaking of every staff and student ${ }^{1}$ - which is a more or less self-contained theme with its own dedicated website; and (2) the Vice Chancellor's blog, as the site for the new Vice Chancellor to communicate with staff at a critical phase in the institution's history.

From each domain, two key videos were selected for the analysis reported on in this article: the 'Senior Leaders Conference Overview' video² (Video 1, Figure 1(a), top), which showcases a two-day event where '[o]ver 130 senior staff across the university met to foster collegiality, share information about current strategic activities and to participate in the Living our Values initiative'; and the 'Meet Curtin University's new Vice-Chancellor: Prof Deborah Terry' video ${ }^{3}$ (Video 2, Figure 1(b), bottom), which was the first video posting on the Vice Chancellor's blog, under the heading 'My background and goals'. This blog has been a primary channel for the incoming Vice Chancellor to communicate with staff, particularly within the context of the comprehensive and dramatic restructure of academic and non-academic staff that was initiated shortly before the new appointment commenced. 


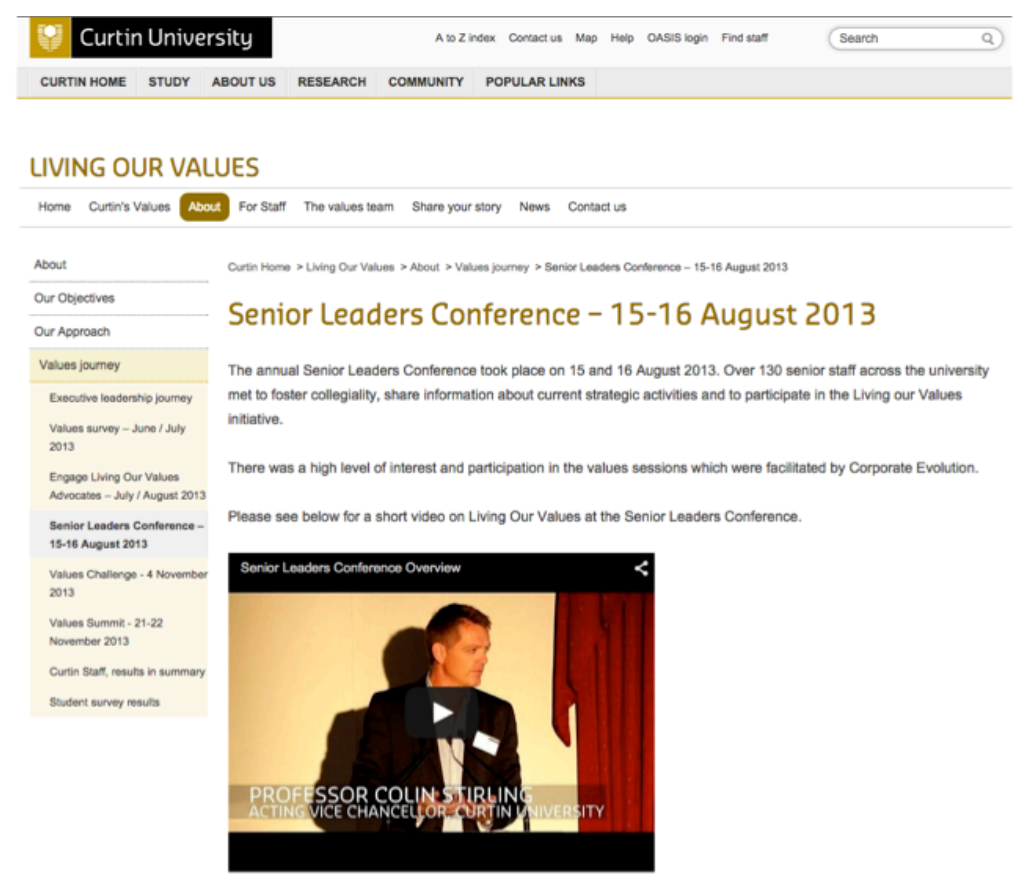

Figure 1. (a)

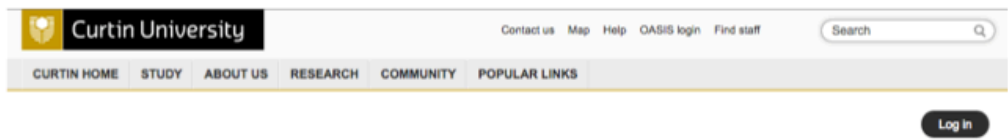

VICE-CHANCELLOR

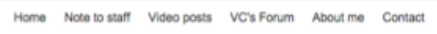

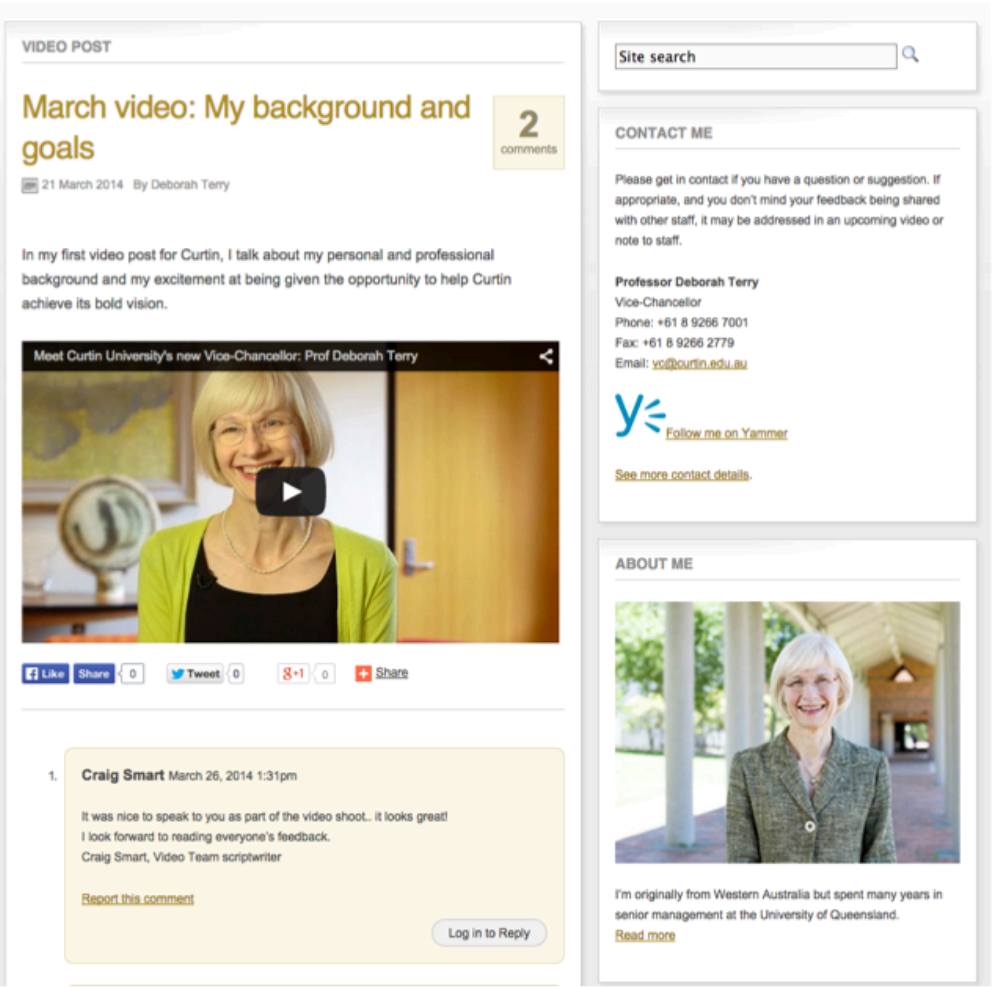

Figure 1. (b)

Figure 1. (a) 'Senior Leaders Conference Overview' (Video 1); and (b) 'Meet Curtin University's new Vice-Chancellor' (Video 2). 
As the analysis will show, the two video texts exhibit markedly different approaches to leadership: Video 1, a professionally produced video of a university event, shows signs of a managerial approach to leadership, whereas Video 2, a seemingly low-cost production of a face-to-face interview with the new Vice-Chancellor, provides evidence of a more collegial style.

\section{The approach: social semiotics and software-based multimodal discourse analysis}

As Taylor and Robichaud (2004: 409) observe, one cannot analyse 'language without reference to context, or context without reference to language'. An important corollary of this is that language in use provides evidence of higher-level contextual phenomena - in this case leadership approaches reflected in online communication. To build a holistic picture on multimodal meaning making in context, the present study adopts a methodology that combines social semiotic theory with software-based tools and techniques.

Social semiotic theory has been a significant influence in multimodal discourse analysis, which studies the contributions and interactions of linguistic and non-linguistic modes (e.g. spoken and written language, image, gesture, sound, page layout and website design) in the communication of meaning. Most social semiotic approaches to multimodal discourse analysis are informed by Halliday's systemic functional theory (e.g. Halliday 1978; Halliday and Matthiessen 2013), which theorises language as a network of systems which are functional in context. Language users make choices from these systems; choices which serve ('realise') the social needs of the discourse participants. The multimodal perspective follows Halliday and Hasan (1985: 4) view, which perceives of context and culture 'as a set of semiotic systems, a set of systems of meaning, all of which interrelate'. To account for the contributions and interactions of linguistic and non-linguistic modes (e.g. spoken and written language, image, gesture, sound, page layout and website design) in the communication of 
meaning, a multimodal discourse perspectives integrates concepts from a diverse range of disciplines such as linguistics, semiotics, film studies, and musicology (e.g. Bateman 2014; Bateman and Schmidt 2011; Kress and Van Leeuwen 2006 [1996]; Jewitt 2014; Machin 2007; Machin and Richardson 2012, O’Toole 2011 [1994]; Van Leeuwen 1999, 2005, 2012).

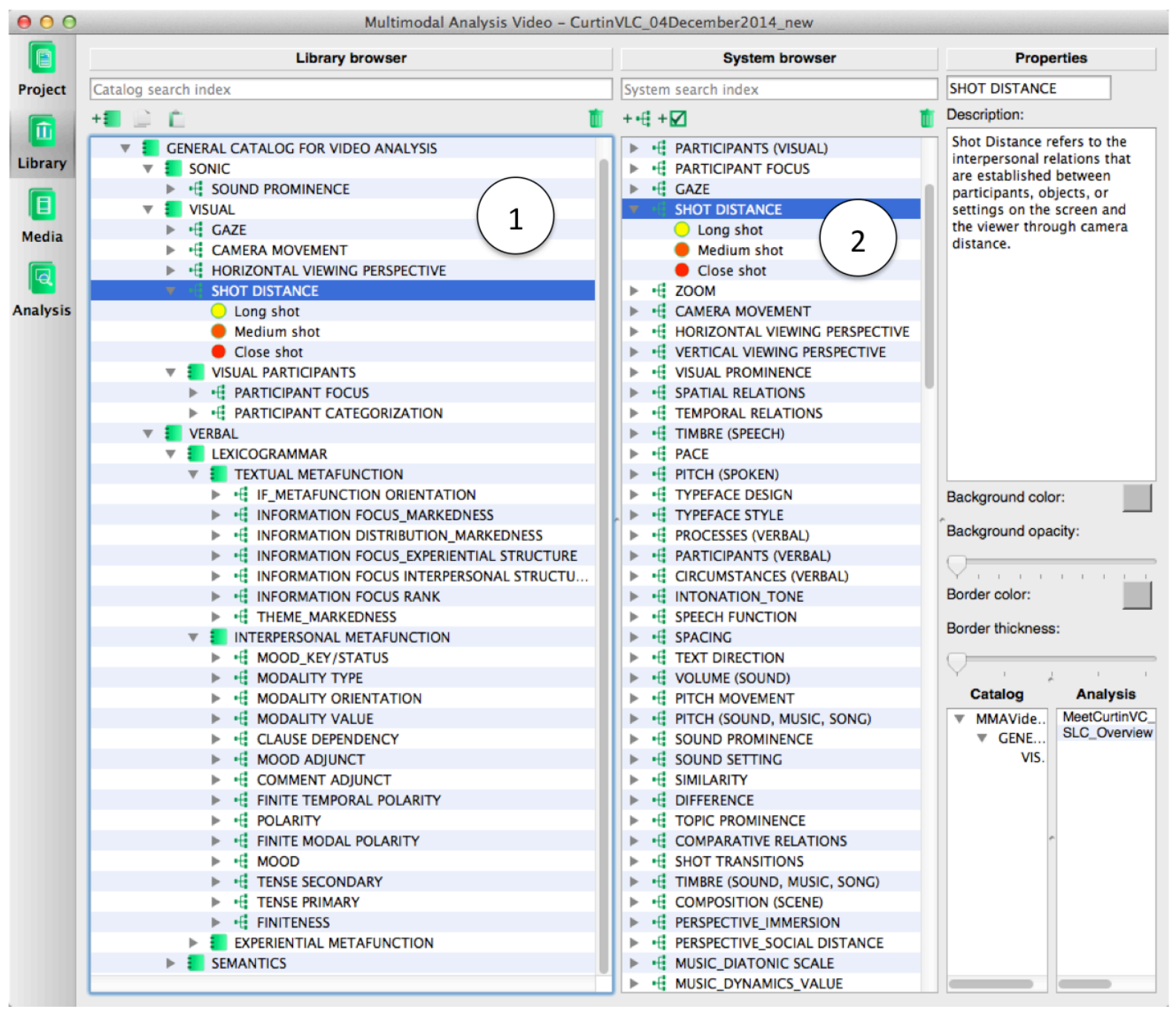

Figure 2. Screenshot of multimodal systems [1] and system choices [2] as displayed in the Systems Library in Multimodal Analysis Video.

The digital multimodal discourse perspective presented in this paper combines multimodal social semiotic theory with software-based techniques to demonstrate (a) how meaning arises from combinations of choices, and (b) how the results the analysis can be related to broader contextual aspects. The approach arose out of the design, development and 
use of an interactive software application for the multimodal analysis of video texts, Multimodal Analysis Video ${ }^{4}$. The software-based (manual) analysis undertaken in this study is supported by the application of integrated system frameworks informed by concepts from social semiotics, systemic functional linguistics, critical multimodal discourse analysis, and film theory (e.g. Bordwell and Thompson 2012; Halliday and Matthiessen 2013; Kress and Van Leeuwen 2006 [1996]; Machin 2007, Machin and Mayr 2012; O’Halloran 2004; O’Toole 2011 [1994]; Van Leeuwen 2005, 2008), to facilitate the identification of key systems used for meaning making in the two videos. For example, the system of SHOT DISTANCE $^{5}$ (see Figure 2), which functions to enact interpersonal relations such as closeness and distance, consists (minimally) of the system choices of Long shot, Medium shot, and Close shot (for further elucidation, see section 'Selected multimodal systems and their contribution to meaning making in context' below). A snapshot of the range of multimodal systems and system choices used for the full analysis of the two video texts discussed in this paper, is displayed in Figure 2.

The videos are annotated manually in a separate GUI, as displayed in Figure 3(a), which provides facilities for viewing the video in the player window [1], and inserting timestamped annotation nodes [2] in system strips [3], by selecting a system choice [4] from the list of available system choices [5] based on the systems created in the Systems Library (Figure 2). All annotations are synchronised with the video player, the film strip [6], the sound strip [7], and the verbal transcription [8]. 


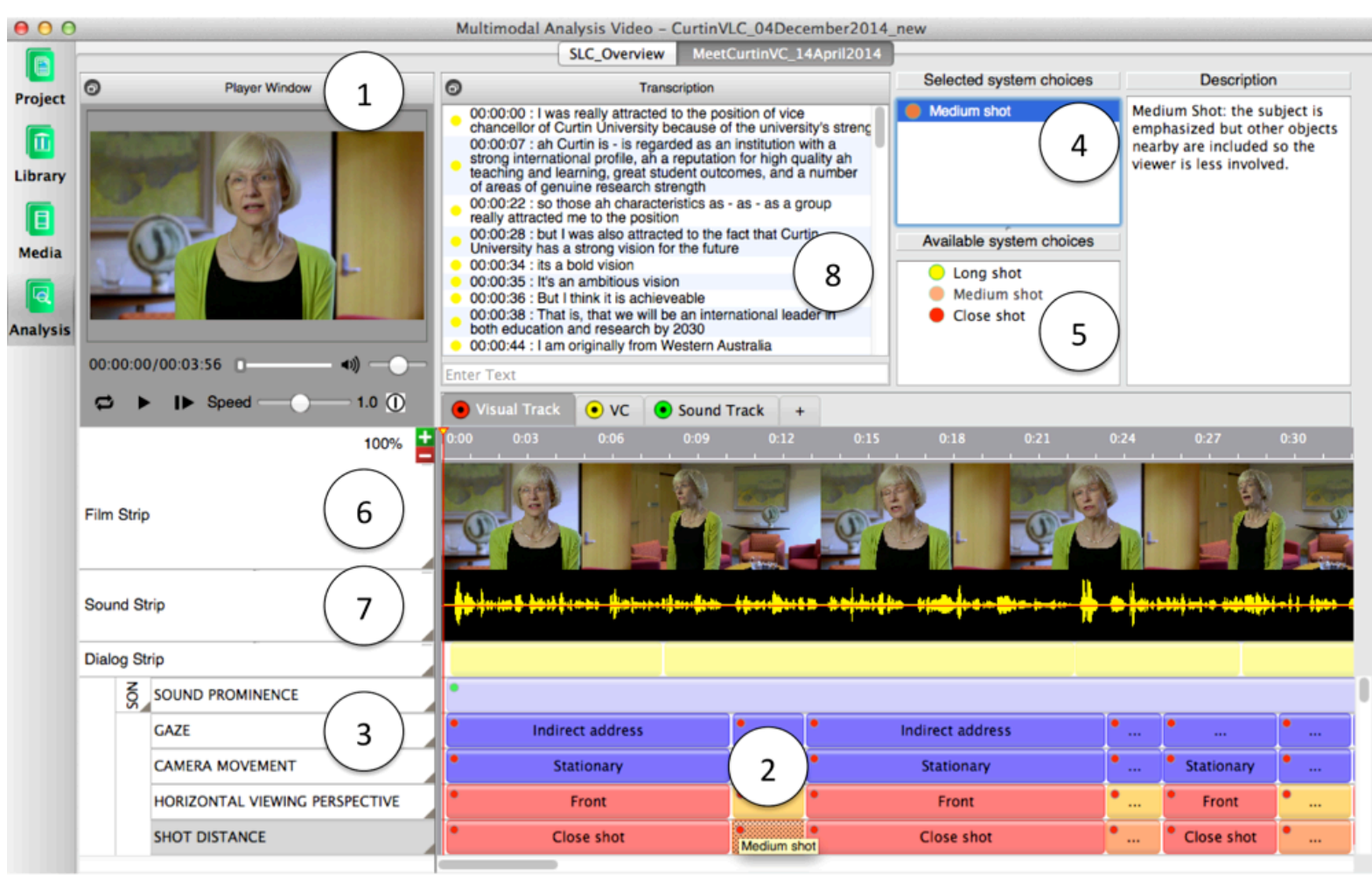

Figure 3. (a)

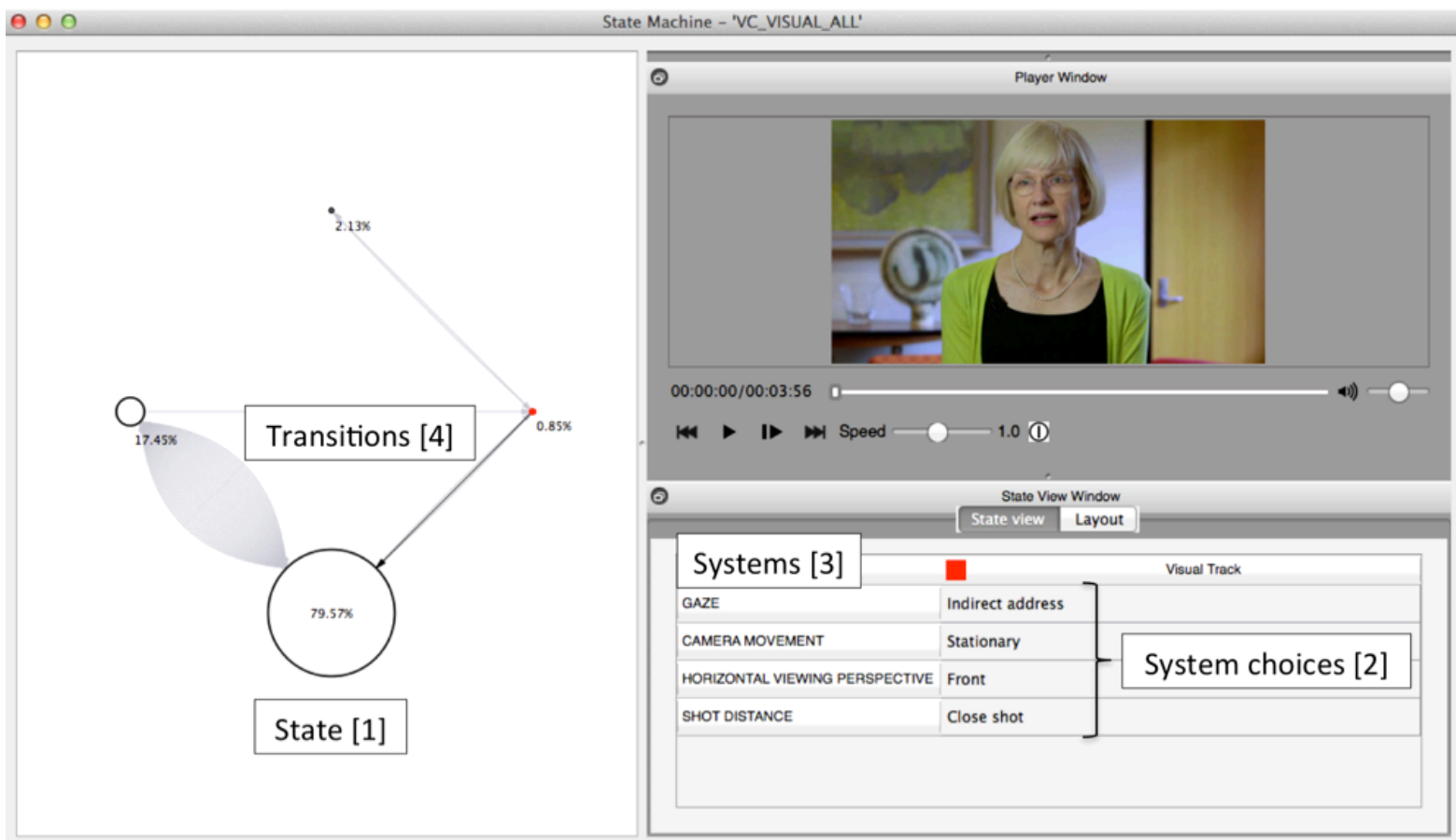

Figure 3. (b)

Figure 3. (a) Screenshot of Video 2 viewed in the Analysis GUI in Multimodal Analysis

Video: player window [1], time-stamped annotations [2], system strips [3], selected system choice [4], list of available system choices [5], film strip [6], sound strip [7], transcription of verbal text [8]; (b) Screenshot of state transition diagram of select visual systems deployed in 
Video 2: state [1], combination of system choices in a state [2], selected systems in the analysis [3], transitions between states [4].

The software provides the additional option to visualise the analysis in the form of state transition diagrams (Figure 3(b)), whereby a 'state' (denoted by circles) [1] represents the system choices [2] that have been utilised for a particular system or a combination of semiotic systems [3], displayed in terms of total video time. The lines between the states represent the movement (i.e. 'transitions') [4] between individual states as the video unfolds. For example, the state diagram in Figure 3(b) reveals that visual meaning in Video 2 is realized predominately through the combination of system choices of Indirect address, Stationary camera movement, Frontal perspective, and Close shot within the systems of GAZE, CAMERA MOVEMENT, HORIZONTAL VIEWING PERSPECTIVE and SHOT DISTANCE, respectively; i.e. State 1, which is deployed in intervallic episodes $79.5 \%$ in terms of total video time (for a discussion of additional information obtained from state diagrams, refer to section 'Analysis and Discussion', 'Visual and sonic systems'). The state transition diagrams thus offer a comprehensive overview of how semiotic choices (linguistic, visual and aural) combine in videos, permitting key systems to be identified and compared across different video genres.

\section{Selected multimodal systems and their contribution to meaning making in context}

Central to social semiotic theory is the concept of 'metafunction', that each instance of text realises three strands of meaning simultaneously: (a) experiential and logical meaning which construes our experience of the world and the logical relations between such meanings; (b) interpersonal meaning which enables us to negotiate social relations and express attitudes; 
and (c) textual or compositional meaning which organises meanings into coherent messages relevant to their context.

Having conducted the full analysis of multimodal systems shown in Figure 2, not all systems utilised were found to contribute to meaning making in context in equal measure. Using both the analysis interface and visualisation facility offered by the software, the systems that were most active and showed most variation in the two videos were determined. The relevance of these systems to the present discussion of leadership approaches manifested in online communication is discussed below.

Visual and sonic systems. The analysis presented below concentrates on combinations of system choices that are drawn upon in visual and sonic data to enact interpersonal relations, given the focus on senior leaders' engagement with staff and other stakeholders. The systems focussed on in the present discussion are: GAZE (direct address, indirect address, no address), CAMERA MOVEMENT (stationary, pan, tilt, pedestal, dolly), HORIZONTAL VIEWING PERSPECTIVE (front, angled, profile, behind, panoramic), SHOT DISTANCE (long shot, medium shot, close shot) and SOUND PROMINENCE (background sound, foreground sound, none).

GAZE, also known as Visual Address, functions to establish interpersonal relations between participants on the screen and the viewer. Viewers will feel more engaged if the participants depicted on the screen are gazing directly at the camera and by extension the viewer, and less engaged if visual participants are not looking directly at the camera. In the first instance viewers are situated as addressees or direct participants in the discourse, while in the second instance viewers are positioned as onlookers or observers.

SHOT DISTANCE and HORIZONTAL VIEWING PERSPECTIVE both function to signify social relations of interpersonal distance and involvement. For example, if participants are framed in a close shot, viewers are likely to feel more involved than if they 
are framed in a medium shot, which conveys social relations that are more formal; whereas long shots which offer a full view of the scene have the effect of creating maximal distance, whereby the viewer is positioned as a spectator. Similarly, shots taken from a frontal perspective typically signal involvement, whilst angled, profile, and panoramic shots tend to signal detachment.

CAMERA MOVEMENT and SOUND PROMINENCE primarily fulfil a textual or compositional function in that they contribute to create an overall sense of rhythm; however they can also work to facilitate a sense of interpersonal involvement. A stationary camera, for instance, concentrates the viewer's attention on the displayed image, while dolly shots, where the camera travels alongside the represented participants, create the sensation of moving along with them. Viewers tend to feel interpersonally more engaged in these shots than in pan, tilt and pedestal shots, where the camera is positioned horizontally and vertically in relation to the represented scene or participant, presenting the diegetic world from the perspective of the viewer as an observer.

Choices made in SOUND PROMINENCE likewise have the capacity to direct viewer attention to particular aspects of the video text. In terms of acoustic salience, foregrounded sounds - 'Figure' in Van Leeuwen's (1999) terms - are sounds 'which the listener must identify with, and/or react to and/or act upon' (Van Leeuwen 1999: 23) and which can create a montage effect (O'Halloran et al. 2011) for accompanying visuals; whereas backgrounded sounds - 'Ground' and 'Field' - tend be perceived as a form of accompaniment, setting, or 'aural wallpaper' (Van Leeuwen 1999: 112) for the visual or verbal discourse thus foregrounded.

Intonational systems. The analysis presented below also focuses on choices in intonational systems in speech. These systems help realise both interpersonal and textual meanings (and also logical meanings, which however are not a point of focus here). These intonational 
choices, not available in writing, enable speakers, even in monologic discourse, to create a greater sense of engagement with their audience through added interpersonal force, and through emphasising particular elements of their discourse, giving those elements added textual significance. The association of such meanings with spontaneous dialogue creates a more personalised, conversational type of text, as opposed to a more formal 'written-like' discourse (Halliday 1985), helping to overcome limitations traditionally associated with formal, monologic, written discourses (i.e. lack of engagement, a sense of 'distance'). In the present research, the analysis thus provides evidence of leadership style, for example in this case, the distinction between a more managerial (hierarchic, 'distant') approach and a more collegial (personal, 'one-to-one') approach. The intonational systems analysed in this research, drawn from Halliday's description (cf. Halliday and Greaves 2008), are KEY, INFORMATION DISTRIBUTION and INFORMATION FOCUS.

KEY systems, manifested in speech through tone choices (rising and falling pitch contours and their combinations), make meaning in combination with MOOD choices. For example, in the declarative mood (e.g. 'it is'), there are a range of further choices in terms of which tone choice is made: the 'neutral' key choice (the default) for this mood is a falling pitch contour, labelled 'tone 1'; but speakers may also choose a high rising pitch contour (tone 2), for example, called the 'challenging declarative' key choice; or a rising-falling pitch contour (tone 5), called the 'committed declarative' key. Meanwhile, for a polar interrogative mood choice (e.g. 'is it?'), one may also choose a rising pitch contour (the 'neutral polar interrogative' key) or a falling pitch contour (the 'peremptory polar interrogative' key). Tone choices may also realise choices in the logical metafunction, in the system of STATUS: the tone 4 (falling-rising pitch contour) 'subordinate' choice ('more to come'); and the tone 3 (level-rise) 'coordinate' choice (also known as the 'listing' tone) - these are, however, not a point of focus in the present work. 
Each instance of a pitch contour (tone choice) also realises a choice in INFORMATION DISTRIBUTION, which involves 'chunking' the flow of speech into information units, each of which represents a 'quantum of information' (Halliday and Matthiessen 2013: 115) in the ongoing flow of information. Speakers may thus vary the rate of information flow, highlighting particular parts of their discourse and also adding further opportunities for choices in KEY systems. The neutral choice is one information unit per clause; 'marked' choices (i.e. atypical choices) can be greater or less than a clause. In the system of INFORMATION FOCUS, the occurrence of each pitch contour highlights a particular element in the discourse flow, called the focus of 'New' information', as being textually important. The neutral choice is the final lexical item in an information unit; but speakers are at liberty to highlight any item as focus of New information, and marked choices have additional textual significance (i.e. are thus additionally highlighted). The analysis of these two information systems thus gives an insight into those aspects of a speaker's discourse that are considered by the speaker to be especially important.

In general terms, the analysis of intonational systems shows how a speaker relates to their audience and presents their messages: whether constructing a more formal discourse characteristic of traditional written leadership communication or creating a more interpersonally lively discourse style normally associated with spontaneous, face-to-face casual conversations (cf. Halliday 1985 for a discussion). In the present study, the analysis of such micro-patterns in discourse reveals a distinction between managerial and collegial approaches to higher education leadership, as discussed in the following section.

\section{Analysis and discussion}

Visual and sonic systems 
As shown in Table 1, the two videos exhibit markedly different patterns in the way they deploy key systems for meaning making at the micro-level of discourse, which can be related to the macro-level structures embodied in different leadership styles and approaches. For instance, in terms of the key visual and sonic systems utilised, the professionally produced Video 1 exhibits far greater diversity than Video 2, a simple production of a face-to-face interview, as evidenced by the variety of system choices which have been selected (e.g. see CAMERA MOVEMENT and HORIZONTAL VIEWING PERSPECTIVE in Table 1).

Table 1. Visual and sonic systems utilised in Video 1 and Video 2.

\begin{tabular}{|c|c|c|c|}
\hline \multirow[t]{2}{*}{ Systems } & \multirow[t]{2}{*}{ System Choices } & \multicolumn{2}{|c|}{$\begin{array}{l}\text { System utilization } \\
\text { (\% in terms of total video duration })^{7}\end{array}$} \\
\hline & & Video 1 [3:22] & Video 2 [3:56] \\
\hline \multicolumn{4}{|l|}{ GAZE } \\
\hline & Indirect address & 25.1 & 97.0 \\
\hline & No address & 68.0 & 2.1 \\
\hline & Direct address & 4.9 & 0 \\
\hline \multicolumn{4}{|c|}{ CAMERA MOVEMENT } \\
\hline & Stationary & 52.0 & 99.2 \\
\hline & Dolly: Sideways & 23.9 & 0 \\
\hline & Dolly: Forward & 2.9 & 0 \\
\hline & Pan: Continuing & 14.1 & 0 \\
\hline & Pan: To a stop & 2.0 & 0 \\
\hline & Pedestal & 4.4 & 0 \\
\hline & Tilt & 1.5 & 0 \\
\hline \multicolumn{4}{|c|}{$\begin{array}{l}\text { HORIZONTAL VIEWING } \\
\text { PERSPECTIVE }\end{array}$} \\
\hline & & 37.0 & 81.8 \\
\hline & Front & & \\
\hline & Angled & 21.7 & 17.4 \\
\hline & Panoramic & 19.7 & 0 \\
\hline & Profile & 14.3 & 0 \\
\hline & Behind & 5.4 & 0 \\
\hline \multicolumn{4}{|c|}{ SHOT DISTANCE } \\
\hline & Close shot & 45.5 & 79.3 \\
\hline & Medium shot & 39.1 & 19.8 \\
\hline & Long shot & 13.4 & 0 \\
\hline \multicolumn{4}{|c|}{ SOUND PROMINENCE } \\
\hline & Foreground sound & 51.5 & 2.5 \\
\hline & Background sound & 46.5 & 0 \\
\hline & None & 0 & 96.6 \\
\hline
\end{tabular}


In Video 1, the combination of choices in the systems of GAZE, CAMERA MOVEMENT, HORIZONTAL VIEWING PERSPECTIVE, SHOT DISTANCE and SOUND PROMINENCE result in an alternating pattern of either establishing or panoramic shots - of the venue, and participant activities - or of to-camera close-up or middle-distance angled or profile shots of featured speakers (see Figure 4). These shots, in social semiotic terms, help to frame the speakers and participants in terms of 'social distance' (e.g. Kress and Van Leeuwen 2006 [1996]): either as something to be engaged with 'face-to-face' or as an impartial observer (the 'audience perspective'). Although general conference participants are the focus of the camera in $38.6 \%$ of total video time, they remain unidentified. In other words, they are impersonalised, and represented as 'tokens of a type' - i.e. 'staff', rather than as individuals with whom we engage face-to-face. In addition, in terms of SOUND PERSPECTIVE, the panoramic shots of general conference participant ('staff') (Figure 4(a)) are usually accompanied by foregrounded music, thus creating a montage effect that distances the viewer further from the visual representations.

This provides a contrast to the 'cameo' appearances of senior staff members, who are featured for $33.7 \%$ of total video duration, either presented close-up to camera, or in (more distant) profile angled shots (see Figure 4(b)). In contrast to the general staff members, who are 'genericised' and represented as members of collectivised social groups (cf. Van Leeuwen 2008), the senior staff members featured in the cameos are 'personalised', being identified by on-screen captions; while the music, which continues throughout the video, is backgrounded (that is, in Van Leeuwen's 1999 terms the sound shifts from 'Figure' to 'Ground') while they are speaking. 

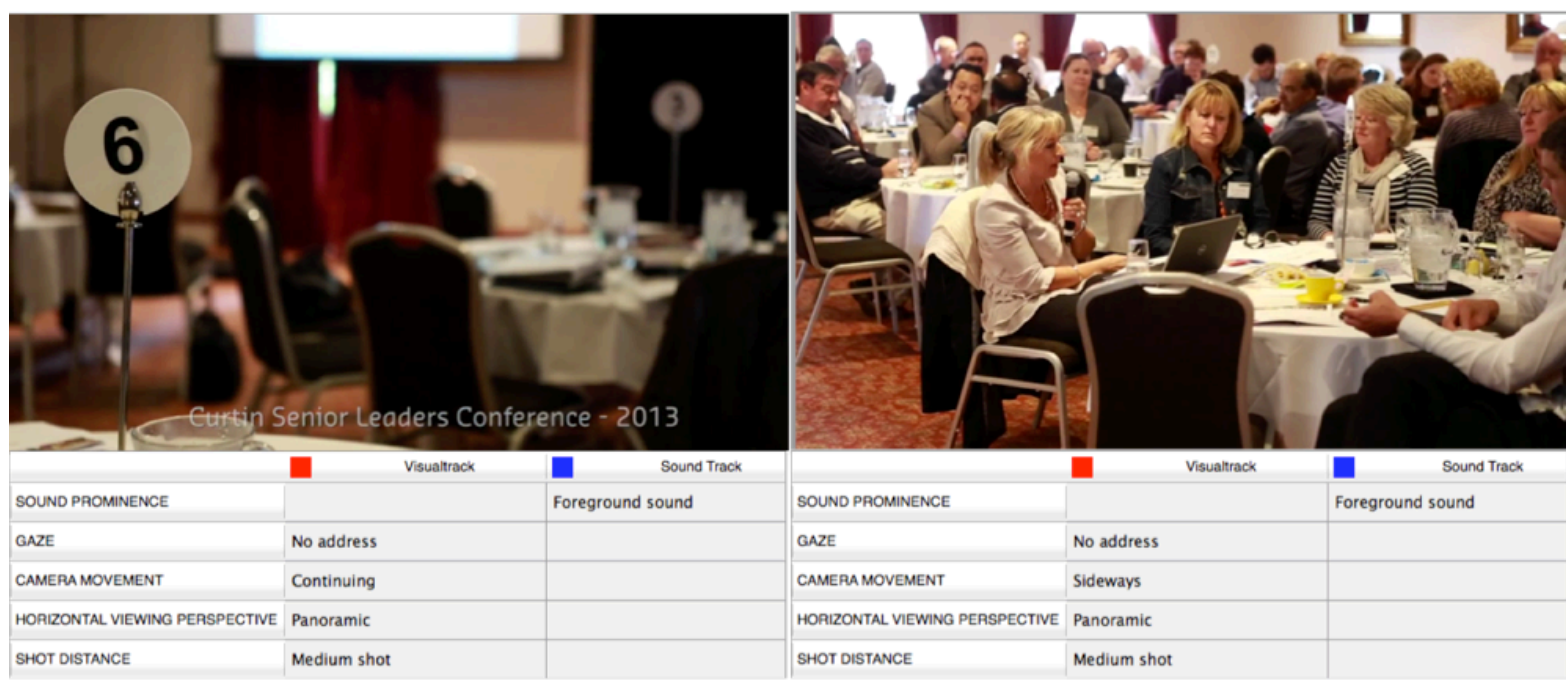

Figure 4. (a)

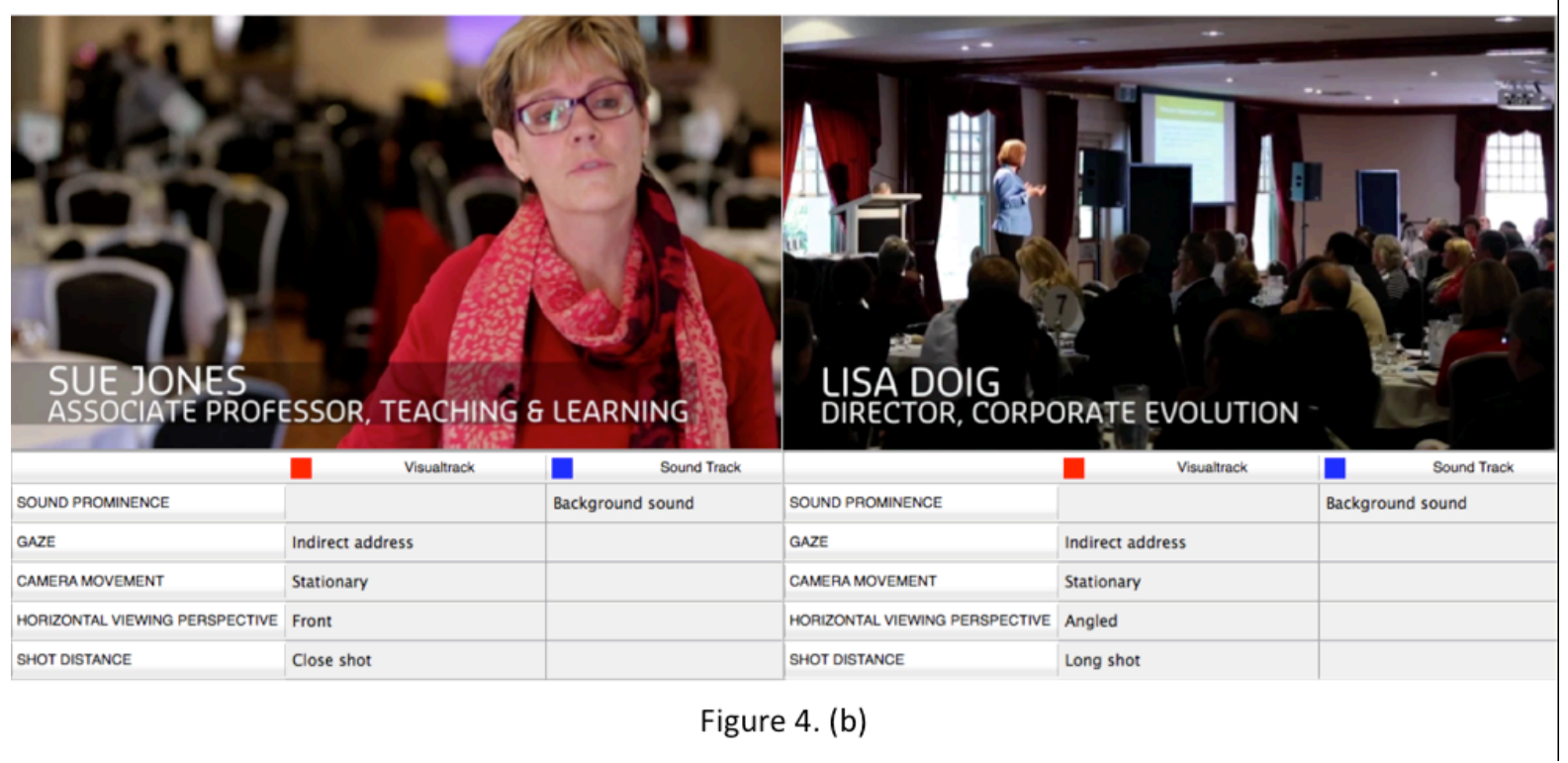

Figure 4. (a) Establishing shot of setting [0:12] (top left), and panoramic shot of participants

$[1: 30]^{8}$ (top right); and (b) close shot of featured speaker [0:39] (bottom left), and long, angled shot of senior executive [1:13] (bottom right).

A peculiarity of Video 1 is that it is constructed out of excerpts from the two-day conference, including the speaker cameos, which are decontextualised, for example missing the relevant prior text (whether prompt or question) to which these cameos appear to be responses. The implication is thus that these short cameos form part of the discourse of a meta-text, the multimodal video itself as text, although with unknown authorship. Following 
Taylor (2011), one could say that these excerpts of 'conversations' with featured academic staff (along with featured discourse of the Acting Vice Chancellor and the session facilitators) are used to construct a 'metaconversation' out of the 'conversations' of the conference. The visual-verbal assemblage of this metaconversation exhibits the characteristics of careful and purposeful editing: for example, while the music plays in the foreground, a distant shot appears of the Acting Vice Chancellor speaking, followed by a closer profile shot [0:49]; then, with a frontal, middle distance shot (he is pictured speaking to the audience), his spoken discourse appears [0:51]: "people um have had an opportunity to really um think about the real the deep reasons why you want to be involved in Curtin: what it is that makes Curtin a great place; um the great things that Curtin does" (for full transcript with mark-up for intonation see Appendix). During this utterance, the visual moves to a series of panoramic shots of staff engaged in discussions and activities during the conference, following the verbal cue of 'people' in the Acting Vice Chancellor's speech [1:02] (see Figure 5).

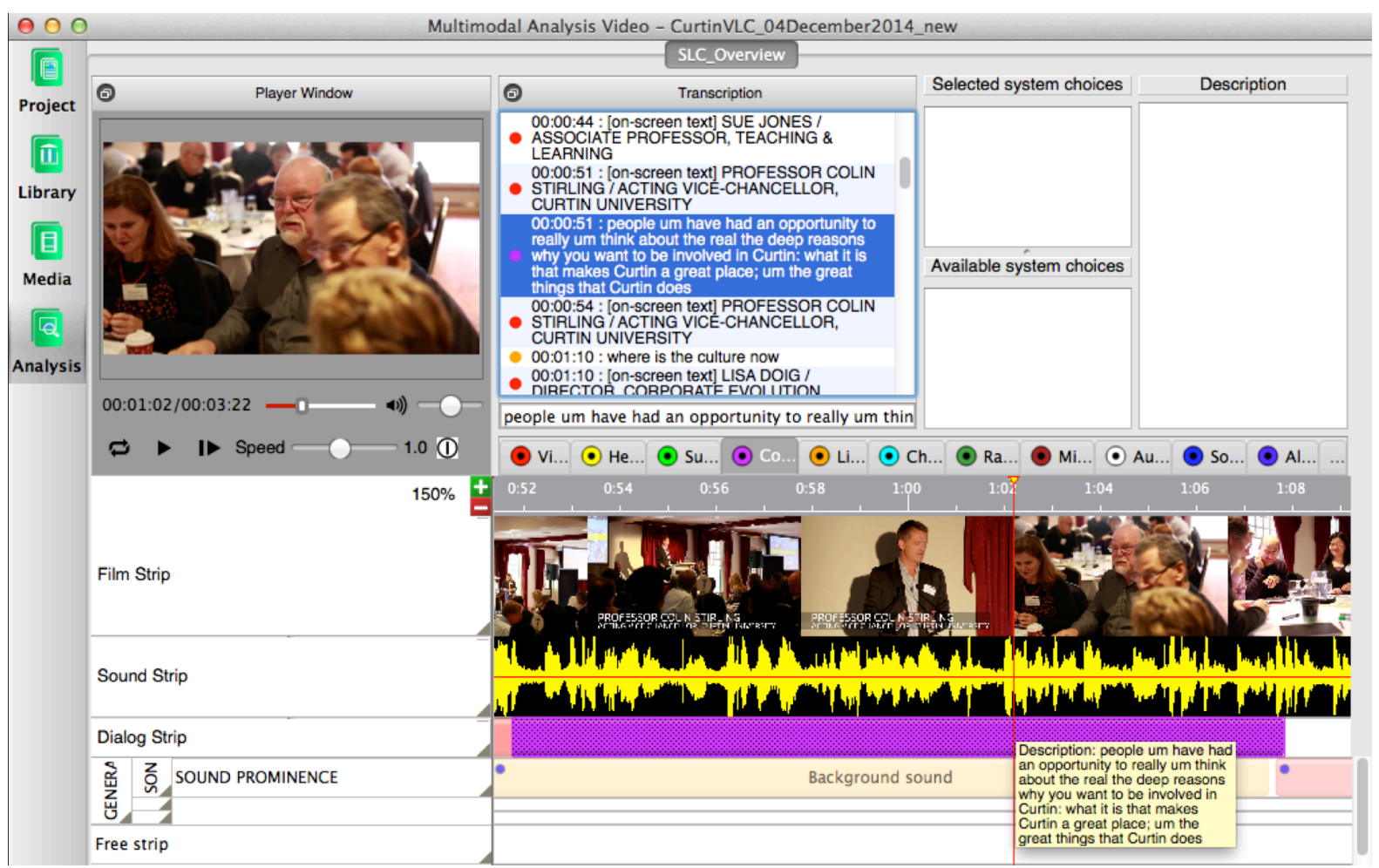

Figure 5. An example of verbal-visual integration. 
In multimodal social semiotic terms, we can say that a verbal-visual logical relation of elaboration is set up: from the Vice Chancellor's utterance 'what it is that makes Curtin a great place' onwards the video depicts visual representations of staff engaged in discussions and activities, which could be interpreted as a visual enactment of their 'involvement' in their institution. Yet here, as elsewhere throughout the video, while staff are represented visually as participating (enacting their commitment to 'being involved' in Curtin'), their verbal discourses are absent. Aside from the featured vignettes of selected senior staff, the only contribution from general staff members is a series of visual shots of written texts, the result of conference participants' group work (and so without individual authorship). Although the status of these material 'objects' (cf. Mersky 2008; Monteiro and Nicolini 2015) - as instances of discourse to be read; or as tokens of a generalisation, i.e. 'staff participation' - is not always clear, in a number of instances $(15.8 \%$ in terms of total video time) these objects are featured in sharp focus, close-up to camera, and thus may be interpreted as 'specified' or 'concreticised' resemiotisations (cf. Van Leeuwen 2008; Iedema and Wodak 1999) of Curtin's values and behaviours (see Figure 6(a)). On the whole, however, Video 1 reinforces the distancing effect between senior management and academics, as observed by Burnes, Wend and By (2013), for example. As such, the visual choices exercised in Video 1 align with a managerial style of executive leadership, which is enacted with what appears to be cooperative and engaged senior staff members. 


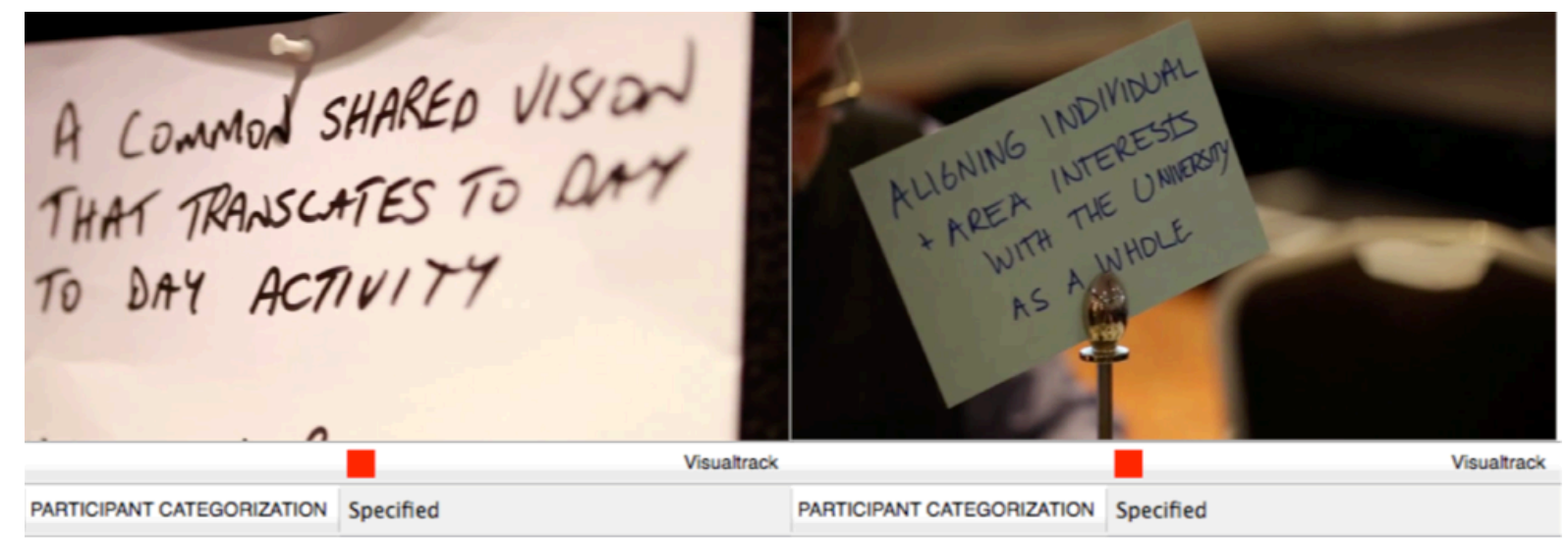

Figure 6. (a)

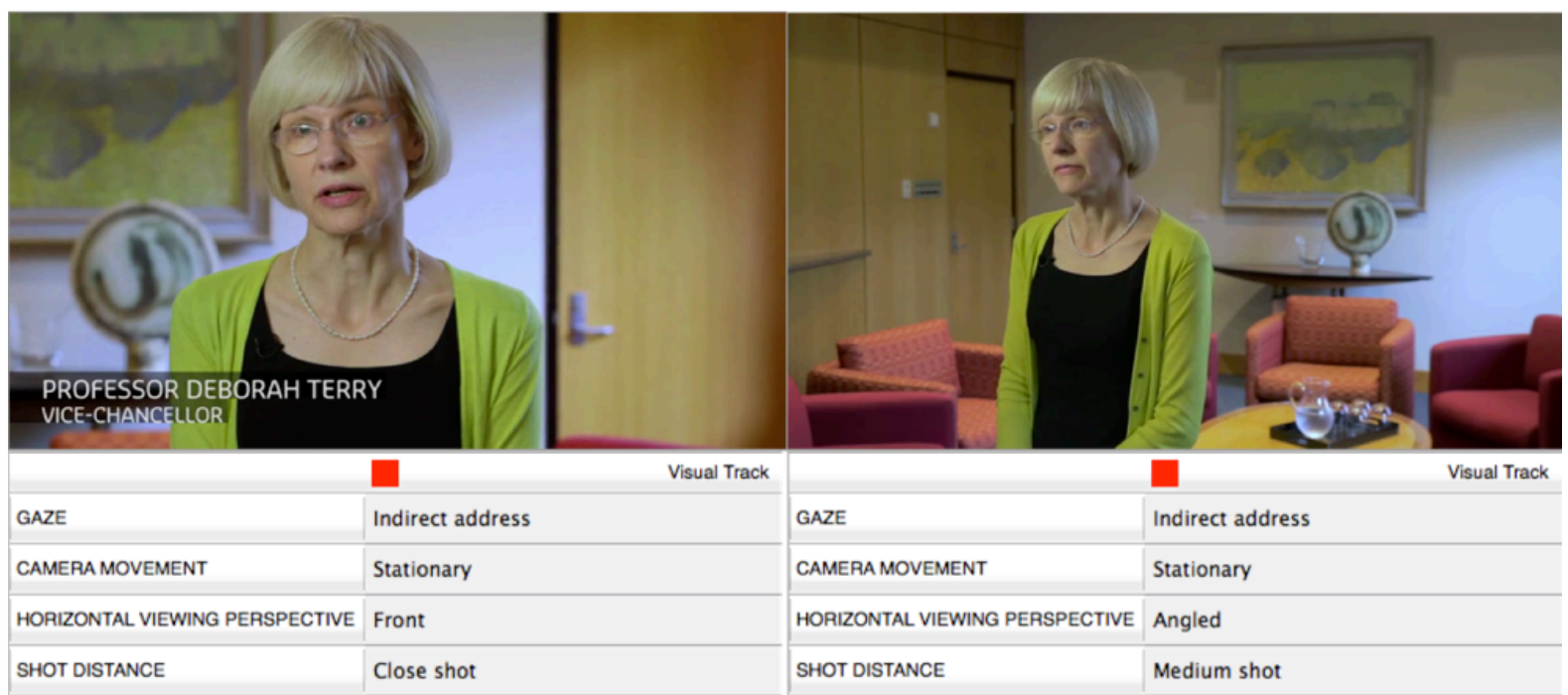

Figure 6. (b)

Figure 6. (a) Resemiotisations of Curtin's values [1:43; $2: 16]$; and (b) the two 'states' of the

Vice Chancellor's visual representations.

In contrast to the diverse meaning-making choices in operation in Video 1, the visual choices utilised in Video 2 are comparatively few (see Table 1) and videographically simple (see Figure 3). The visual track in Video 2 alternates between two distinct 'states': (a) closeup stationary shots of the Vice Chancellor positioned facing in the direction of the camera but not gazing at it directly (79.6\% in terms of total video time), and (b) angled profile shots of the Vice Chancellor at medium distance (17.5\% in terms of total video duration) (see Figure 6(b) for illustration). While these shots appear similar in style to the views of speakers featured in the cameos in Video 1, in that the Vice Chancellor is represented as individualised 
and personalised by being formally identified by means of on-screen captions, there is no musical soundtrack in Video 2. In contrast to the distancing montage effect evident in Video 1, the audience is directly engaged via the absence of music in Video 2. The only views in Video 2 are of the Vice Chancellor who, although positioned gazing in the direction of the camera, never looks at it directly, being clearly pictured as addressing someone off-camera, to the effect that the viewer is positioned as observing (or being present at) the conversation. The combination of choices made in the Vice Chancellor's gaze, calm demeanour, semicasual attire, and the lounge-like setting visible in the background, function to create a relaxed atmosphere.

It is significant that the incoming Vice Chancellor chose to feature her first communication with staff in a video blog interview. This choice can be interpreted as an indication that, for the new Vice Chancellor, it was important to overcome the impersonal, distancing effect of the prototypical 'authoritative' written text from institutional leader, to enact a more strongly collegial approach, presenting herself 'in person' (albeit in mediated form), and drawing on the semiotic resources of facial expression, gaze, body posture, and so forth, and of the spoken voice (e.g. intonational systems), to discuss her 'background and goals'.

\section{Intonational systems}

The following analysis of intonational systems focuses on the percentage frequency of choices (rather than percentage duration for visual and sonic choices and states), derived from an Excel spreadsheet exported from the video analysis software. In Video 1 there are 41 information units, compared to 113 information units in Video 2 (52.2\% of Video 1 discourse being non-verbal). Again, it is found that the two videos exhibit markedly different patterns in the way they utilise KEY choices in intonational systems, which - as the analysis will 
show - can be related to the different leadership styles and approaches evident in the two videos.

Table 2. Intonational choices in Videos 1 and 2.

\begin{tabular}{|c|c|c|c|}
\hline Systems & System Choices & $\begin{array}{l}\text { Video 1: } \\
\text { choices }(\%)\end{array}$ & $\begin{array}{l}\text { Video } 2 \\
\text { choices }(\%)\end{array}$ \\
\hline \multicolumn{4}{|c|}{ KEY/ STATUS } \\
\hline & neutral declarative & 43.9 & 45.1 \\
\hline & subordinate & 29.3 & 12.4 \\
\hline & coordinate & 7.3 & 11.5 \\
\hline & committed declarative & 7.3 & 4.4 \\
\hline & neutral imperative & 4.9 & 0 \\
\hline & neutral wh-interrogative & 2.4 & 0 \\
\hline & neutral + confirmatory declarative & 2.4 & 8.8 \\
\hline & mild declarative & 2.4 & 1.8 \\
\hline & reserved declarative & 0 & 3.5 \\
\hline & $\begin{array}{l}\text { committed }+ \text { confirmatory } \\
\text { declarative }\end{array}$ & 0 & 2.7 \\
\hline & challenging declarative & 0 & 5.3 \\
\hline & confirmatory declarative & 0 & 2.7 \\
\hline & strong declarative & 0 & 1.8 \\
\hline \multicolumn{4}{|c|}{ INFORMATION } \\
\hline \multicolumn{4}{|c|}{ DISTRIBUTION } \\
\hline & unmarked & 61.0 & 46.0 \\
\hline & marked: less than clause & 39.0 & 53.1 \\
\hline & marked: more than clause & 0 & 0.9 \\
\hline \multirow{3}{*}{\multicolumn{4}{|c|}{$\begin{array}{l}\text { INFORMATION } \\
\text { FOCUS: } \\
\text { MARKEDNESS }\end{array}$}} \\
\hline & & & \\
\hline & & & \\
\hline & unmarked & 90.2 & 67.3 \\
\hline & marked: Head & 7.3 & 8.0 \\
\hline & marked: Modifier & 2.4 & 20.4 \\
\hline & marked: Preposition & 0 & 2.7 \\
\hline & marked: Conjunction & 0 & 1.8 \\
\hline
\end{tabular}

As shown in Table 2, Video 2 has a broader spread of choices in KEY than Video 1 (the state transition diagram in Figure 7 below shows the spread of choices for the two videos). As discussed earlier, variation in KEY choices enacts a more lively sense of interpersonal engagement with the listener. In Video 2, the Vice Chancellor uses more marked (i.e. atypical) KEY choices (i.e. those other than neutral KEY choices for each mood), $22.2 \%$ compared to $9.7 \%$ in Video 1 . This pattern of variation and markedness in 
KEY choices gives the impression of a more conversational and personalised type of communication - evidence or a more collegial approach being enacted.

In spite of there being a pattern of more complex clause structures in Video 1 (embedded clauses, complex nominal groups etc), we find a greater percentage of marked INFORMATION DISTRIBUTION choices in Video 2: 39\% in Video 1 compared to 54\% in Video 2 (one choice in the latter is more than one clause, a short conditional clause complex: 'and although we left when I was quite young'). This again suggests a more active use of intonation in Video 2: i.e. the choices of how to 'chunk up' the discourse is motivated by reasons other than clause structure and complexity. Firstly, the assignment of marked INFORMATION DISTRIBUTION enables extra KEY choices; secondly, it also engenders additional choices in INFORMATION FOCUS; both are evident in Extract 1, Video 2 [1:41]: Extract 1

//1 they're always the most difficult questions //4 to answer //

Again, as discussed earlier, the additional KEY choices enabled by marked INFORMATION DISTRIBUTION (here, the tone 1 neutral declarative KEY for the first information unit, "they're always the most difficult questions") add a heightened sense of interpersonal activity to a discourse, thus giving the Vice-Chancellor's discourse a more engaged, personalised feel. This engagement is also evident in the additional choices of focus of New (in this case, marked New, 'difficult' being a non-final lexical item) engendered by a marked choice of INFORMATION DISTRIBUTION, which allows the speaker to add additional highlighting to particular elements of her discourse, drawing the hearer's attention to these in a more persuasive, interactional style. This is further evident in Extract 2, Video 2 [0:08]:

Extract 2 
//53 ah Curtin is - is regarded as an institution with a strong international profile //4 ah a reputation for high quality //3 ah teaching and learning //1 great student outcomes //1 and a number of areas //1 of genuine research //1 strength //

Such added textual emphasis as accorded to the bolded items in Extract 2 is almost entirely absent in prototypically formal leadership discourse, as represented by Video 1.

Tracking patterns of choice in INFORMATION FOCUS gives a valuable insight into the text in terms of those elements in the discourse the speaker has deemed worthy of textual emphasis (one can scan the bolded items in the Appendix to get a sense of this perspective on the texts). The comparison of whether choices in the INFORMATION FOCUS system are marked (e.g. 'difficult' in 'most difficult questions' above) or unmarked (i.e. typical) is particularly significant: whereas $9.7 \%$ of choices in Video 1 are marked, $32.7 \%$ are marked in Video 2. These choices add additional highlighting to the elements upon which the marked focus of New falls, being the non-default choices. Of the Vice Chancellor's marked choices of New, $20.4 \%$ are assigned to a Modifier of a clause group or phrase, as seen in these example excerpts. The pattern of marked highlighting of Modifiers throughout this text can be interpreted within the framework of Appraisal theory (Martin and White 2005): it increases ('graduates') the interpersonal force of these positive appraisals. This, again, provides evidence of the Vice-Chancellor enacting a more lively, interactive style of discourse: she is clearly attempting to fully engage those listening to this spoken discourse through such choices - in particular, it can be assumed, her new colleagues who are the primary audience for the incoming leader's first video blog posting.

These intonational systems, as with the visual and sonic systems discussed above, work together within discourse, as in Extract 3, Video 2 [2:16]:

Extract 3 
$/ / 4$ and I - I guess the third thing //4 ah more seriously //5 is that really passionate about - 'bout universities //

Here, marked choices in all systems enable a textual focus on both the circumstance Adjunct 'the third thing' and the interpersonal (comment) Adjunct 'more seriously' signaling a significant shift in the overall discourse - as well as on the Attribute the speaker assigns to herself, 'passionate', together with the marked committed declarative KEY (tone $5)$.

In summary, whilst the intonational choices exercised by the participations in Video 1 are characteristic of more formal, 'written-like' discourse types such as formal speeches, showing less variation in interpersonal and textual meanings, the analysis of intonational choices in the Vice Chancellor's spoken discourse in Video 2 shows a more lively use of interpersonal and textual systems, thus revealing a more conversational and personalised discourse style. Accordingly, the analysis of Video 1 provides evidence of a more formal, managerial approach to leadership discourse, whilst the patterns of choice in intonational systems in Video 2 suggest an attempt at enacting a more collegial approach to engaging staff and other potential audiences by the new Vice-Chancellor.

\section{Putting it all together: a critical multimodal perspective on leadership discourses}

The above analysis reveals distinct patterns of choice and variation between the two videos, which appear to correspond with different approaches to leadership embodied in each. This variation in use of visual, sonic and verbal systems is illustrated in the four state transition diagrams in Figure 7, showing the spread of choices in selected intonational and visual systems, represented as 'states' or combinations of choices, together with the 'transitions' (i.e. shifts) between those states. The visualisations in Figure 7 clearly show that visual systems play a much more active meaning making role in Video 1 (Figure 7, top left), a 
carefully produced and edited multimodal document ( $52 \%$ of Video 1 is non-verbal communication), than they do in the recorded face-to-face interview with the Vice Chancellor in Video 2 (Figure 7, bottom left; see also visualisation in Figure 3(b)), but that in the latter intonational choices (Figure 7, bottom right) play a more active role in the enactment of a personalised, collegial approach.

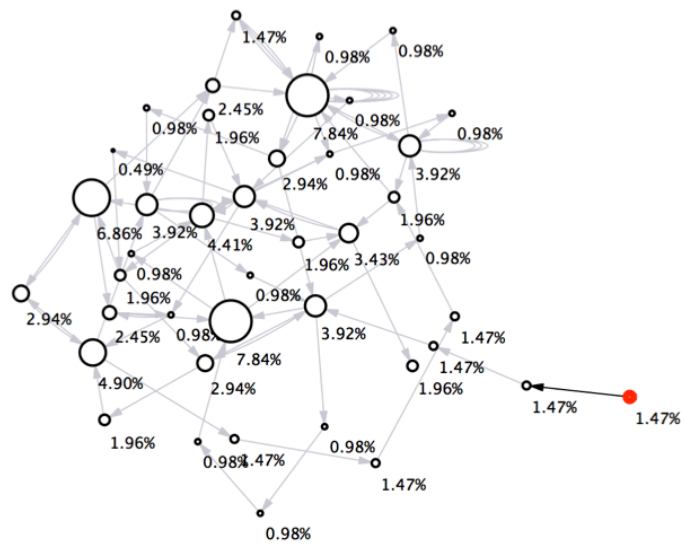

Video 1 Selected Visual Systems

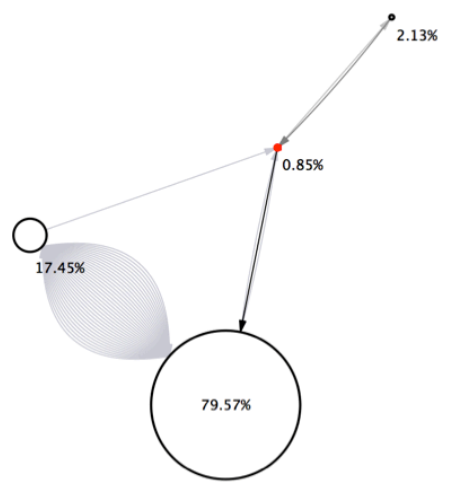

Video 2 Selected Visual Systems

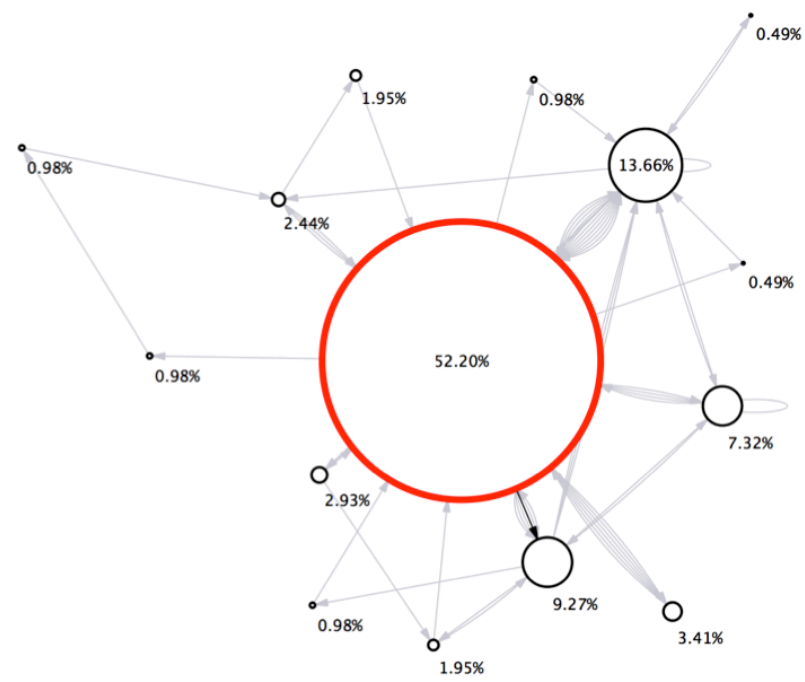

Video 1 Selected Intonation Systems

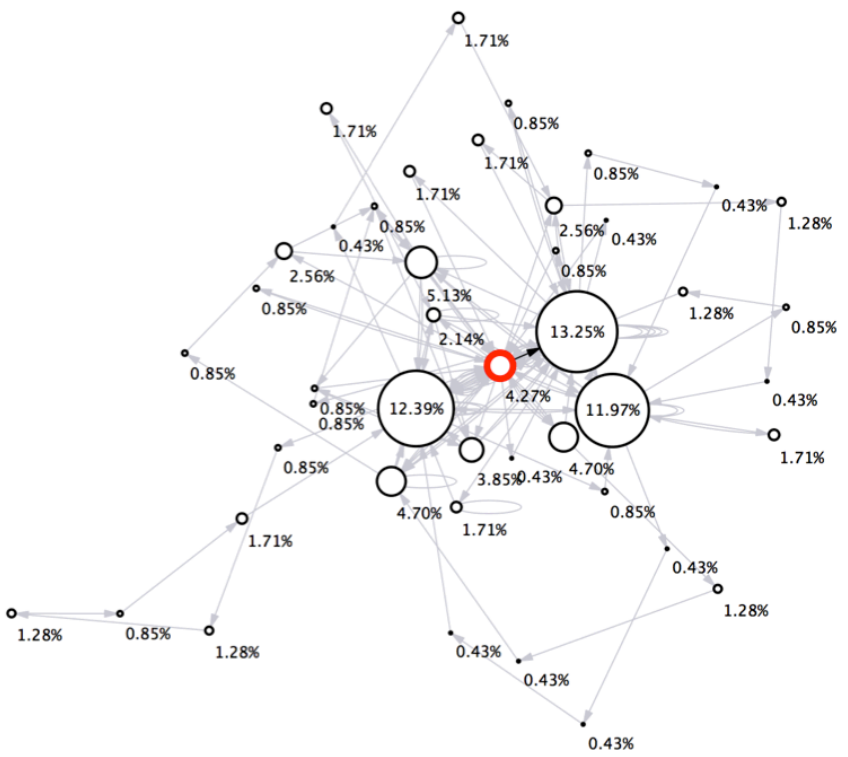

Video 2 Selected Intonation Systems

Figure 7. State transition visualisation of diversity in selected visual and intonation systems in Video 1 (top) and Video 2 (bottom). 
The above micro-level analysis of multimodal choices reveals how such discourses are the embodiment of the macro-level 'institutional logics' discussed earlier (those of managerialism and collegialism); and shows how, with online mediation, the nature of leadership communication is changing. Rather than formal, written proclamations of policy, important institutional 'authoritative texts', as discussed by Blaschke, Frost and Fabian (2014), are no longer written documents only. As the above analysis has shown, they are just as likely to be mediated in the form of a carefully produced multimodal video text, or couched in the relatively informal, conversational style of a video blog. Yet, multimodal documents such as the two video texts analysed in this study are clearly meant to be authoritative documents - the fact that they are public in itself suggests this.

The online mediation of leadership communication however problematises distinctions noted in the literature on traditional leadership communication, for example between authoritative texts and distributed conversations (Blaschke, Frost and Fabian 2014). Although clearly representing the distributed conversations of staff, the depersonalisation evident in Video 1 recalls Taylor's (2011: 1278) claim that 'the actual maker or author of the text, may vanish from view, be made absent, with the result that it is the text that now becomes invested with authority...'. For example, the 'Living our Values' initiative featured in Video 1 seems itself clearly intended to engage staff in a collegial, consultative process. Indeed, the inclusion of featured cameos of academic staff insinuates a collegial approach, indicating staff participation. However, this representation falls short of the authoritative voice assigned to executive leadership and their agents. In Video 1, it is clearly the Acting Vice Chancellor, selected senior staff members and the corporate change facilitators (who are also featured prominently in the video) who are given authoritative semiotic agency. They represent the 'voice' to be attended to, hence privileging a managerial approach to leadership. 
Conversely, online mediation also opens up possibilities for more interactive, personalised and collegial styles, as evidenced by the communicative systems deployed by the Vice Chancellor in Video 2, where the combination of multimodal choices invokes a sense of a personal 'conversation' with staff, in spite of this being, as one of the first public documents from the new Vice Chancellor, an authoritative text.

\section{Conclusions, limitations and future research}

The above analysis has shown that empirical evidence afforded by computational tools and techniques can be related to higher level, abstract (humanist) interpretations of multimodal texts. As the analysis and discussion above makes clear, with the online mediation of university leadership discourse, the study of such texts from a digital multimodal discourse perspective becomes crucial. Such texts, as public documents, invite closer scrutiny than the unrecorded discourses traditionally associated with the negotiation of values and other meanings between staff and leadership. As the above analysis of the two video texts has shown, new approaches are required to study the affordances of multimodal discourses that are enabled by online, digital media. The article has demonstrated that a digital multimodal discourse perspective facilitates the identification of key multimodal systems and their interactions to create meaning in discourse; and that detailed manual analysis of micropatterns in discourse can offer insights into larger sociocultural patterns related to different leadership styles and approaches as manifested in on-line communication, providing a digital-empirical basis for traditionally discursive humanist discourse interpretation.

The case study presented in this article is part of a larger research project concerned with developing digital, multimodal approaches for the study of online corpora, involving extensive analyses of text and images from the 'Living our Values' and Vice Chancellor blog websites, as well as Twitter discourse within the same university campus. Constraints of 
space and focus has meant that it has been possible to present only a small portion of these analyses, to illustrate the digital multimodal perspective with a focus on online leadership approaches. Clearly, a focus on other important multimodal resources for meaning making, such as gesture, would reward further research.

The present discussion is intended to show the need for further research into online multimodal discourses, and also the development of digitally-enabled approaches capable of relating detailed, complex multimodal phenomena to broader sociocultural contexts, as embodied, for example, in changing communicative practices in higher education. In an online age, leaders, here as elsewhere in society, are recognising that online communication is central to modern leadership styles and approaches. But as university leaders are given more scope for multimodal communication through online mediation, they also face the challenges of greater complexity in such discourses. As scholars, if we are to grasp the nature of contemporary leadership discourse in higher education and its challenges, we need to study these discourses as multimodal communication. Moreover, the digital age is presenting changes at such a rapid pace that the challenge for scholars of discourse and communication is to keep stride with such developments. To adequately account for the increasingly multimodal discourses in the public, online realm, new approaches are needed, particularly those that exploit the immense potential of digital software as a tool for discourse analysis.

\section{Funding}

The authors thank Curtin University for providing funding for the 'Mapping Discourses of Values, Leadership and Policy at Curtin University' pilot project.

\section{Notes}

1 http://www.curtin.edu.au/livingourvalues/ 
2 http://www.curtin.edu.au/livingourvalues/about/senior-leaders-conference-15-16-august-2013.cfm

3 http://blogs.curtin.edu.au/vice-chancellor/video-posts/2014/march-video-post/

4 http://multimodal-analysis.com/products/multimodal-analysis-video/

5 It is a convention in Systemic Functional Linguistics (SFL) to use all upper caps for system names.

6 It is a convention in SFL for structural labels to have upper caps initial.

7 Figures are rounded up or down to one decimal point; hence percentages do not always add up exactly to $100 \%$

8 Time in minutes and seconds of actual videos

\section{References}

Bateman J (2014) Looking for what counts in film analysis: a programme of empirical research. In: Machin D (ed) Visual Communication. Berlin: De Gruyter Mouton, pp. 301-329.

Bateman J and Schmidt KH (2011) Multimodal Film Analysis: How Films Mean. London and New York: Routledge.

Blaschke S, Frost J and Hattke F (2014) Towards a micro foundation of leadership, governance, and management in universities. Higher Education 68(5): 711-732. DOI: 10.1007/s10734-014-9740-2.

Bordwell D and Thompson K (2012) Film Art: An Introduction (10th edition). New York: McGraw-Hill.

Bryman A (2007) Effective leadership in higher education: a literature review. Studies in Higher Education 32(6): 693-710. DOI: 10.1080/03075070701685114.

Burnes B, Wend P and By RT (2013) The changing face of English universities: reinventing collegiality for the twenty-first century. Studies in Higher Education 39(6): 905-926. DOI: $10.1080 / 03075079.2012 .75485$. 
Cooren F, Kuhn T, Cornelissen JP and Clark T (2011) Communication, organizing and organization: an overview and introduction to the special issue. Organization Studies 32(9): 1149-1170. DOI: 10.1177/0170840611410836.

Fairclough N (2005) Discourse analysis in organization studies: the case for critical realism. Organization Studies 26(6): 915-939.

Fullan M and Scott G (2009) Turnaround Leadership for Higher Education. San Francisco: John Wiley \& Sons.

Halliday MAK (1978) Language as Social Semiotic. London: Edward Arnold.

Halliday MAK (1985) Spoken and Written Language. Waurn Ponds, Victoria: Deakin University Press.

Halliday MAK and Hasan R (1985) Language, Context and Text: Aspects of Language in a Social-Semiotic Perspective. Waurn Ponds, Victoria: Deakin University.

Halliday MAK and Greaves WS (2008) Intonation in the Grammar of English. London: Equinox.

Halliday MAK and Matthiessen CMIM (2013) An Introduction to Functional Grammar (3rd edition). London: Arnold.

Iedema R (2001) Analysing film and television: a social semiotic account of Hospital: an unhealthy business. In Van Leeuwen T and Jewitt C (eds) Handbook of Visual Analysis. London; Thousand Oaks; New Delhi: Sage Publications, pp. 183-204.

Iedema R and Wodak R (1999) Introduction: organizational discourses and practices. Discourse Society (10)5: 5-19.

Jewitt C (ed) (2014) The Routledge Handbook of Multimodal Analysis (2nd edition). London: Routledge.

Kress G and Van Leeuwen T (2006 [1996]). Reading Images: The Grammar of Visual Design (2nd edition). London: Routledge. 
Kuhn T (2012) Negotiating the micro-macro divide: thought leadership from organizational communication for theorizing organization. Management Communication Quarterly 26(4): 543-584. DOI: 10.1177/0893318912462004.

Lemke JL (2002) Travels in hypermodality. Visual Communication 1(3): 299-325.

Machin D (2007) Introduction to Multimodal Analysis. London: Hodder Arnold; New York: Oxford University Press.

Machin D (2013) What is multimodal critical discourse studies? Critical Discourse Studies 10(4): 347-355.

Machin D and Mayr A (2012) How to Do Critical Discourse Analysis: A Multimodal Introduction. SAGE Publications.

Machin D and Richardson JE (2012) Discourses of unity and purpose in the sounds of fascist music: a multimodal approach. Critical Discourse Studies (9)4: 329-345.

Marginson S (2010) How universities have been positioned as teams in a knowledge economy world cup. In: Blackmore J, Brennan M and Zipin L (eds) Re-positioning University Governance and Academic Work. Netherlands: Sense Publishers, pp.1734.

Martin JR and White PRR (2005) The Language of Evaluation, Appraisal in English. Palgrave Macmillan, London and New York.

Mersky RR (2008) Lost in transition: the use of role objects in today's postmodern organizations. Organisational and Social Dynamics 8(1): 97-112.

Middlehurst R and Elton L (1992) Leadership and management in higher education. Studies in Higher Education 17(3): 251-264. DOI:10.1080/03075079212331382527.

Monteiro P and Nicolini D (2015) Recovering materiality in institutional work: prizes as an assemblage of human and material entities. Journal of Management Inquiry 24(1): 61-81. 
O'Halloran KL (ed) (2004) Multimodal Discourse Analysis. London: Continuum.

O’Halloran KL (2011) Multimodal discourse analysis. In: Hyland K and Paltridge B (eds) Companion to Discourse Analysis. London: Continuum, pp.120-137.

O'Halloran KL, Tan S, Smith BA and Podlasov A (2011) Multimodal analysis within an interactive software environment: critical discourse perspectives. Critical Discourse Studies 8(2): 109-125.

O’Mullane M (2011) University Leadership: Approaches, Formation and Challenges in Europe. Basingstoke; New York: Palgrave Macmillan.

O’Toole M (2011 [1994]) The Language of Displayed Art (2nd edition). London/New York: Routledge.

Scott G, Bell S, Coates H and Grebennikov L (2010) Australian higher education leaders in times of change: the role of Pro Vice-Chancellor and Deputy Vice-Chancellor. Journal of Higher Education Policy and Management 32 (4): 401-418.

Tapper T and Palfreyman D (2010) The Collegial Tradition in the Age of Mass Higher Education. Dordrecht: Springer.

Taylor JR (2011) Organization as an (imbricated) configuring of transaction. Organization Studies 32(9): 1273-1294.

Taylor JR and Robichaud D (2004) Finding the organization in the communication: discourse as action and sensemaking. Organization 11(3): 395-413.

Van Dijk TA (2001) Multidisciplinary CDA: a plea for diversity. In Wodak R and Meyer M (eds) Methods of Critical Discourse Analysis. London: Sage, pp. 95-120.

Van Leeuwen T (1999) Speech, Music, Sound. Houndsmills: Macmillan Press.

Van Leeuwen T (2005) Introducing Social Semiotics. London: Routledge.

Van Leeuwen T (2008) Discourse and Practice: New Tools for Critical Discourse Analysis. New York: Oxford University Press. 
Van Leeuwen T (2012) The critical analysis of musical discourse. Critical Discourse Studies, 9(4): 319-328. 


\section{Appendix: Transcript with intonational systems}

Note: double forward slashes show the division into information units; the numeral indicates the tone choice; bold type indicates the focus of New (including major+minor double focus for the compound tones 13 and 53).

\section{Video 1}

Speaker 1: //1 our biggest challenge in shifting our values //1 is in how we communicate it effectively // Speaker 2: //1 I think the biggest challenge that we have ahead of us in looking at the values at Curtin University is having the courage //1 to have the type of conversations that we need to have // Speaker 3: //4 people um have had an opportunity to really um think about the real the deep reasons why you want to be involved //1 in Curtin //3 what it is that makes Curtin a great place //5 um the great things that Curtin does // Speaker 4: //3 where is the culture now //4 where do you desire to be //4 what's in the way //1 and ah you know what would the values and behaviours be to shift // Speaker 5: //1 I've worked with a couple of companies that have gone through a similar process that Curtin's going through at the moment //1 with introducing values $/ / 4$ and the biggest challenge I think //3 is leadership //1 and effectively showing staff the way // Speaker 6: //1 Collaboration //4 cooperation //4 communication //1 information sharing //__ (mild) that would actually work // Speaker 4: //4 so how do you have that dynamic balance between //1 all the different stakeholders you have //5 and its also about finding an identity //1 you know what is Curtin's identity //5 the values speak to the identity // Speaker 3: //13 Our staff say we want accountability right //1 we want accountability //4 we really want //1 accountability //1 so let's - let's give our staff accountability //4 let's all be accountable // Speaker 7: //4 I'm going to motivate others //4 to live their values //1 by living mine //1 My values are around inclusivity and communication //1 and I will be demonstrating those in everything that I do // Speaker 3: //1 If we can cascade that through the organisation //4 I think we can have a really - the - the people and culture project here //1 can have a really profound impact // 


\section{Video 2}

Speaker 1: //1 I was really //4 attracted to the position of vice chancellor of Curtin University //1 because of the university's //1 strengths //53 ah Curtin is - is regarded as an institution with a strong international profile //4 ah a reputation for high quality //3 ah teaching and learning //1 great student outcomes //1 and a number of areas //1 of genuine research //1 strength //3 so those ah characteristics //3 as - as - as a group // really attracted me to the position $/ / 1+$ (strong) but I was also attracted to the fact that $/ / 3$ Curtin University has a strong vision for the future //4 its a bold vision //4 It's an ambitious vision //1 But I think it is achieveable //1 That is, that we will be an international leader //1 in both education and research //1 by $2030 / / 13 \mathrm{I}$ am originally from Western Australia //13 um my father's family is from the Margaret River area //1 and although we left when I was quite young //3 we spent a lot of our holidays here //13 and so its always - it was a very important part of - of my childhood //1 and ah its very nice to be back in Western Australia //13 My early impressions of Curtin //1 are very very positive $/ / 3$ ah everybody has been incredibly welcoming $/ / 3$ which has been very nice //1 and it is very clear //53 that staff are hugely committed to what they do at Curtin $/ / 1$ and - and - and passionate about //1 the institution //1 and about their work //2 There is a lot of change going on //2 at the moment // and as I've said at a number of different forums already //2 I'm very supportive //2 of the change //4 I think ah the changes that are in train //2 are - are necessary //4 if we are to achieve our strategic goals //1 Nevertheless //1 the changes have to be implemented very carefully //4 and very thoughtfully //1 and I'm putting a lot of time in at the moment //1_(mild) to ensure that that is the case //13 Three things about myself //1 they're always the most difficult questions //4 to answer //1 ah but I can tell you //13 that my ah husband and children would probably say //13 that one thing ah people need to know //3 is that I'm no good if I haven't had coffee in the mornings //1 so I've been very pleased to find ah very good coffee //2 on campus already //4 ah second thing //53 I'm a - a sort of enthusiastic cyclist //1 ah so we've um really been pleased to discover all of the cycle tracks //1 around Perth //3 so really enjoy getting out on the weekend //4 and ah having a look around //1 clearing my head //1 after a busy week //4 and I - I guess the third thing //4 ah more seriously //5 is that really passionate about - 'bout universities //1 Universities are very important //1 institutions //1 and ah you know I really enjoy engaging //1 with staff //1 and alumni //1 and it - and working with staff //5 it's just amazing //5 ah how $\mathrm{mu}[\mathrm{ch}]$ - what diverse things happen at universities //1 and how committed people are //1 to what they're doing //1 I think what I bring to Curtin University //1+3 (strong) is the fact that I've um held a number of different roles //1 at another large comprehensive and 
research intensive //1 university in Australia //1 and I think that experience //1 combined //5 with the talent that is here //1 already //13 ah at Curtin //1 will mean we are //1 well-positioned //1 to meet our strategic objectives $/ / 1$ as - as - as an institution //3 I hope that people realise that - that I am accessible //1 that I'm really open //4 (reserved) to feedback and ideas //1_ (mild) but at the same time //3 I will be driven by clear plans for the future //1 I do like //3 to see the evidence //3 and I'm - I'm data driven //4_ (reserved) in terms of my decision-making //5 but ov[er] - the overriding goal //4 is that I am driven by excellence // 1 and impact // 1 in everything we do //3 I've already received a lot of feedback from staff //3 and I'm looking forward to receiving more comments //4_(reserved) ideas through you know many different forums //3 I see these video posts as being um a regular ah feature that I'll be involved in //4 and obviously //1 will continue as much as I can to get out and about // and meet people // 


\section{Author biographies}

Dr Sabine Tan is Research Fellow at the School of Education, Faculty of Humanities, Curtin University. Her research interests include critical multimodal discourse analysis, social semiotics, and visual communication. She has worked on interdisciplinary projects involving the development of multimodal analysis software for research and educational purposes.

Dr Bradley A Smith completed his PhD research at Macquarie University, and has since researched and taught at National University of Singapore (Research Fellow), University of Melbourne (Tutor in Higher Education) and Curtin University (Research Fellow). His research interests include the study of intonation in language, multimodality (with a focus on sound) and register variation (with a focus on the roles and relations of speech and writing).

Dr Kay O’Halloran is Associate Professor, School of Education, Faculty of Humanities, Curtin University. Her areas of research include multimodal analysis, social semiotics, mathematics discourse, and the development of interactive digital media technologies and mathematical and scientific visualization techniques for multimodal and sociocultural analytics. 